\title{
Les révolutions à l'épreuve du marché
}

Leora Auslander, Charlotte Guichard, Colin Jones, Giorgio Riello et Daniel Roche

Traducteur : Julien Louvrier

\section{OpenEdition}

Journals

Édition électronique

URL : https://journals.openedition.org/ahrf/12525

DOI : $10.4000 / a h r f .12525$

ISSN : 1952-403X

Éditeur :

Armand Colin, Société des études robespierristes

Édition imprimée

Date de publication : 1 décembre 2012

Pagination : 165-190

ISBN : 978-2-200-92762-2

ISSN : 0003-4436

\section{Référence électronique}

Leora Auslander, Charlotte Guichard, Colin Jones, Giorgio Riello et Daniel Roche, « Les révolutions à

l'épreuve du marché », Annales historiques de la Révolution française [En ligne], 370 | octobre-décembre 2012, mis en ligne le 01 décembre 2015, consulté le 22 avril 2022. URL : http://

journals.openedition.org/ahrf/12525; DOI : https://doi.org/10.4000/ahrf.12525 
REGARDS CROISÉS

\title{
LES RÉVOLUTIONS À L'ÉPREUVE DU MARCHÉ
}

\author{
Leora AUSLANDER \\ Charlotte GUICHARD \\ Colin JONES \\ Giorgio RIELLO \\ Daniel ROCHE
}

Le marché fait-il les révolutions ? Telle est la question que nous avons posée à des historiens de la consommation et du marché, de la culture matérielle, des révolutions. Les révolutions : la formule s'entend au sens large, et désigne non seulement les révolutions politiques (en France et sur le continent américain), mais aussi les révolutions industrielles et ce qu'un nombre croissant d'historiens appellent la révolution de la consommation. Depuis les travaux de Daniel Roche en France, de Neil McKendrick et John Plumb en Angleterre, l'histoire de la consommation s'est considérablement développée et la marchandisation de la société est devenue un trope dans les études dix-huitiémistes, même si les notions de marché, de « révolution de la consommation » et la figure du consommateur restent encore largement discutées entre spécialistes. Dans cette perspective d'histoire économique, les rapports entretenus entre ces mutations de la consommation et les révolutions industrielles ont fait l'objet de nouvelles interrogations, notamment dans l'ouvrage de Jan De Vries ${ }^{1}$.

Tout ce courant historiographique est resté assez imperméable aux interrogations que soulèvent les révolutions politiques de la fin du

(1) Jan de VRIES, « The Industrial Revolution and the Industrious Revolution », Journal of Economic History, juin 1994, 54/2, p. 249-70 ; id., The Industrious Revolution: Consumer Demand and the Household Economy, 1650 to the Present, Cambridge, Cambridge University Press, 2008. Voir la note critique de Jean-Yves GRENIER, " "Travailler plus pour consommer plus". Désir de consommer et essor du capitalisme, du XVII ${ }^{\mathrm{e}}$ siècle à nos jours », Annales. Histoire, Sciences Sociales, 2010/3, p. 787-798. 
XVIII ${ }^{\mathrm{e}}$ siècle, en dépit des travaux d'Éric Hobsbawm ${ }^{2}$. Certains historiens saluent certes l'articulation accrue entre histoire du marché et histoire des révolutions politiques : l'article de Colin Jones « The Great Chain of Buying » qui démontrait les liens intimes entre les transformations du marché et les formes de la Révolution française conserve à cet égard toute son importance. Mais d'autres au contraire soulignent que l'historiographie de la consommation - par ailleurs longtemps restée suspecte aux yeux des historiens de l'économie - reste encore réfractaire à toute dimension politique, selon l'argument qu'elle serait cantonnée à l'histoire de la sphère domestique. De fait, on a là deux courants historiographiques traditionnellement séparés, entre une histoire industrielle et économique centrée sur l'Angleterre, et une histoire politique des révolutions centrée sur la France et les Amériques.

La rencontre de ces deux historiographies se pose d'autant plus que chacune est désormais prise dans une vision qui s'est globalisée. L'histoire économique des révolutions industrielles et de la consommation s'est mondialisée et intègre maintenant le continent asiatique ${ }^{3}$, tandis que l'ouvrage récent dirigé par David Armitage et Sanjay Subrahmanyam témoigne d'un élargissement de l'historiographie des révolutions politiques dans une perspective véritablement mondiale ${ }^{4}$.

Ces historiographies se sont donc globalisées et leur rencontre se pose de plus en plus, non plus dans la perspective marxiste mais dans des termes qu'il convient d'inventer.

Dans leurs travaux sur les origines de la Révolution industrielle, les historiens de l'économie ont longtemps privilégié le XIXe siècle. Depuis maintenant une trentaine d'années, le XVIII siècle a pris une part extrêmement importante dans ces études à la fois dans le monde anglophone, sous l'impulsion de John Plumb, Roy Porter et John Brewer, et en France, avec les travaux de Daniel Roche. On a souvent opposé ces deux approches : l'une serait davantage inscrite dans une généalogie de la culture de masse et de la naissance de la société de consommation, tandis que l'autre s'intéresserait

(2) Eric J. HoBSBAWM, The Age of Revolution : Europe 1789-1848, Londres, Weidenfeld et Nicolson, 1962.

(3) Kenneth Pomeranz, The Great divergence : China, Europe, and the making of the modern world economy, Princeton, Princeton University Press, 2000 ; Giorgio RIELLO, Cotton: The Fabric that Made the Modern World, Cambridge, Cambridge University Press, à paraître.

(4) David ARMitage et Sanjay Subrahmanyam (éd.), The Age of Revolutions in Global Context, c. 1760-1840, Farnham (GB), Palgrave Macmillan, 2010. 
davantage à une anthropologie du quotidien et à une histoire de la culture matérielle dans une tradition braudélienne. Dans quelle mesure ces distinctions historiographiques ont-elles encore un sens? Que nous disent-elles des objets et des méthodologies proposées? Comment le XVIII siècle s'inscrit-il dès lors dans un récit de la modernité ?

\section{Daniel Roche}

Le déplacement de l'intérêt pour la Révolution industrielle du XIX au XVIII ${ }^{\mathrm{e}}$ siècle, et de surcroît celui de sa place dans les origines de la Révolution française, masque un débat ancien sur l'existence et la datation d'une révolution industrielle, car tous les historiens de l'économie ne sont pas d'accord sur les ruptures décisives. Qualitativement, avec Patrick Verley, je pense qu'une vraie rupture se situe entre 1760 et 1870, sans sous-estimer les changements intervenus dans les économies préindustrielles. L'enjeu de la question me paraît double. Le premier est à replacer dans l'idée de comprendre les facteurs explicatifs de la transformation, de ce point de vue mettre l'accent sur la naissance d'une société de consommation aboutit à rassembler les préalables du développement, et ainsi, à justifier le décollage par le choix d'une variable. D'autre part, la combinaison des causes est soumise à interprétation. Donc mon intérêt pour la société de consommation ne vise pas à relier le temps de la longue durée (la culture matérielle braudélienne) à la formation de la culture des masses consommatrices comme facteur unique, ce qui est difficile à démontrer à l'échelle seule de l'Europe comme on le voit dans le modèle de l' Industrious Revolution proposée par Jan de Vries ${ }^{5}$. Il s'agit plutôt de comprendre comment des relations nouvelles se nouent entre les différents éléments du système social, culturel et économique. Ce qui est le second enjeu des études associant l'analyse du changement politique, les Révolutions, avec celle des modifications de l'Ancien Régime économique dans son fonctionnement et dans son interprétation. En bref, quand j'ai commencé à travailler pour le Peuple de Paris, j'essayais de comprendre, comme Michelet et Labrousse, ce qui comptait de la pauvreté et de la richesse. Quand je l'ai achevé, j'avais conscience d'interroger un changement fondamental des comportements hiérarchisés selon les capacités et les nouveaux besoins, avec de nouvelles sensibilités, ceci dans une économie où l'agriculture 
progressait peu, mais où la culture et la circulation urbaine intensifiaient une demande de nouveaux produits, étalaient la visibilité des consommations des vrais riches, accéléraient la demande des classes moyennes et des riches de la pauvreté.

\section{Colin Jones}

Si l'on considère que l'on a longtemps vu la Révolution française comme l'origine de la culture politique moderne, l'attention portée au XVIII ${ }^{\mathrm{e}}$ siècle dans les récits de la modernité n'est pas franchement chose nouvelle. Toutefois, au cours des dernières décennies, en portant une attention plus grande aux origines intellectuelles de la Révolution, les historiens se sont aventurés plus loin dans le XVIII ${ }^{\mathrm{e}}$ siècle. Ils ont trouvé sur ce terrain des historiens du social, de la culture et des idées qui avaient eux-mêmes trouvé à s'entendre pour défricher un champ laissé vierge, du moins en Grande-Bretagne, de toute incursion historiographique des historiens de l'économie.

De ce point de vue, ce sont les travaux pionniers devenus classiques de Neil McKendrick sur le comportement des consommateurs au XVIII ${ }^{\mathrm{e}}$ siècle qui retiennent l'attention. Au lieu de supposer, comme auraient eu tendance à le faire d'après lui la plupart des historiens de la Révolution industrielle, qu'une offre améliorée suscite invariablement une demande prise pour argent comptant et très insuffisamment problématisée, McKendrick part de l'hypothèse inverse que la demande féminine de biens manufacturés au cours du XVIII ${ }^{\mathrm{e}}$ siècle est à l'origine de l'apparition d'une offre renouvelée ${ }^{6}$. La publication de The Birth of a Consumer Society, écrit par McKendrick avec John Plumb et John Brewer en 1982, a montré à quel point cette « découverte » de la demande s'est révélée fructueuse en permettant aux historiens de repenser des aspects primordiaux de l'histoire sociale, culturelle et politique.

Au moment même où cette nouvelle approche est venue chambouler le milieu des spécialistes de l'histoire anglaise du XVIII siècle, Daniel Roche faisait la démonstration de son utilité auprès des dix-huitièmistes français. Là où McKendrick s'était confronté au poids de l'historiographie de la Révolution industrielle en Grande-Bretagne, Roche s'attaquait lui à

(6) Neil MCKENDRICK, « Home demand and economic growth : a new view of women and children in the industrial revolution », Neil MCKENDRICK (ed.), Historical Perspectives: Studies in English Thought and society in Honour of J.H. Plumb, Cambridge, 1974. Voir également l'article publié par Margot Finn qui reprend l'argument d'une façon intéressante, « Men's things : masculine possessions in the consumer revolution », Social History, 25, 2000. 
une lecture de l'économie française au XVIII ${ }^{\mathrm{e}}$ siècle dominée alors par l'arithmétique sociale convaincante de son maître, Ernest Labrousse. La paupérisation signalée par Labrousse est dure. Avec une augmentation des prix agricoles de $62 \%$, des salaires de $26 \%$, une baisse du niveau de vie de $15 \%$ à $25 \%$, l'équation laisse peu de place à la surprenante abondance d'objets matériels que Roche et ses élèves ont commencé à recenser dans les maisons, les placards et les armoires des classes populaires parisiennes au cours du dernier siècle de l'Ancien Régime. En France, on avait jusqu'alors tenu la consommation pour un phénomène de la fin du $\mathrm{XIX}^{\mathrm{e}}$ siècle voire $\mathrm{du} \mathrm{XX}^{\mathrm{e}}$ siècle. En la faisant remonter au XVIII ${ }^{\mathrm{e}}$ siècle, le livre de Daniel Roche, Le Peuple de Paris, paru en 1981, a fait l'effet d'un pavé dans la mare jusqu'à provoquer quelques dommages collatéraux et involontaires en faisant exploser quelques-unes des conceptions majeures de l'interprétation jacobino-marxiste de la Révolution française. Il était ainsi approprié que les classes populaires avant 1789 - les futurs « sans-culottes »-portent des culottes et non le pantalon des travailleurs que les sans-culottes ont adopté ensuite.

Comme le suggère cet aperçu un peu rapide, l'historiographie de la société de consommation a joué un rôle catalyseur vital en modifiant la place accordée au XVIII ${ }^{\mathrm{e}}$ siècle dans les discours de la modernité. L'intérêt historiographique grandissant pour le comportement des consommateurs au XVIII siècle a profondément modifié les deux grands discours de la modernité existants, constitués autour de la Révolution industrielle en Grande-Bretagne et autour de la Révolution française en France. Par conséquent, le XVIII ${ }^{\mathrm{e}}$ siècle dans son ensemble a bénéficié d'une attention bien plus importante que ce n'était le cas jusque là.

Enfin, il faut noter que malgré la capacité du modèle consumériste à remettre en cause les paradigmes existants, les différences au sein des cadres nationaux de la recherche sur la place du XVIII ${ }^{e}$ siècle dans les discours de la modernité sont au moins aussi importantes que les différences entre les écoles historiques nationales. En Angleterre, John Plumb et Roy Porter sont par essence des historiens néo-Whig (une catégorie qui n'a pas d'équivalent en France et qu'il ne faut absolument pas confondre avec les néo-libéraux) qui croient dans la réalité du progrès, quoiqu'en se montrant plus conscients de ses coûts que leurs prédécesseurs Whig. Ceci dit, on ne peut pas réduire l'historiographie britannique à cette approche. De la même façon, on pourrait discerner en germe à la fois dans la tradition des Annales et aussi (malgré les divergences mentionnées plus haut) dans l'œuvre d'Ernest Labrousse, le cadre de longue durée dans lequel Daniel Roche inscrit son travail, qu'il s'agisse du Peuple de Paris ou de sa superbe 
Histoire des choses banales. Naissance de la société de consommation (XVIII ${ }^{\mathrm{e}}$-XIX ${ }^{\mathrm{e}}$ siècles). Toutefois, Roche se garde bien de faire irruption de façon trop intrusive dans l'historiographie française des révolutions politiques de la fin du XVIII ${ }^{\mathrm{e}}$ siècle qui ont toujours attiré à elles une grande partie de l'école historique française.

\section{Giorgio Riello}

L'histoire de la consommation a certainement contribué à mettre le $\mathrm{XVIII}^{\mathrm{e}}$ siècle sur le devant de la scène. Traditionnellement, le domaine de compétence des historiens économiques de la Révolution industrielle s'est exercé sur les six décennies comprises entre 1770 et 1830 , période de transition d'une société moderne, depuis les classiques de l'histoire industrielle, jusqu'aux histoires sociales à la Asa Briggs et aux grands tableaux vivant à la Hobsbawm. Au cours des trois dernières décennies, la consommation s'est imposée irrésistiblement dans l'historiographie comme un outil permettant de poser des problèmes historiques qui sont essentiels à notre identité actuelle. Une partie des méthodes est empruntée à l'histoire économique, mais je pense que c'est l'influence de l'anthropologie et dans une moindre mesure de la sociologie qui a davantage contribué à faire évoluer les questions que se posent les historiens. Pour ce qui est de l'opposition entre une façon britannique («anglo-saxonne») et une façon française (ou « continentale ») d'aborder la consommation au XVIIIe siècle, $j$ ' avoue que je la trouve peu convaincante. L'interprétation de McKendrick, Brewer et Plumb, influencée par l'agenda «néo-libéral » des années 1980 et les idées de liberté et d'individualisme, oublie un peu que McKendrick avait déjà clairement formulé ses arguments au début des années 1960 dans ses travaux sur Josiah Wedgwood. Elle oublie également les nombreuses contributions qui suivirent le livre de McKendrick et Alt et qui n'étaient certainement pas, elles, «néo-libérales ». Personne ne niera l'existence de différences nationales, qu'explique l'utilisation de sources différentes, mais deux livres comme ceux de Daniel Roche, Le peuple de Paris (1981) et celui de Peter Earle, A City Full of People (1994) peuvent suggérer qu'il existe des intérêts communs et des choix méthodologiques partagés. En outre, on a vu paraître au cours des dix dernières années, des travaux sur l'Espagne, les Pays-Bas ou la Scandinavie, pour n'en citer que quelques-uns, qui soulèvent des questions qui ne rentrent ni dans le cadre de la consommation de masse ni dans celui d'une anthropologie du quotidien. La littérature sur le sujet est désormais abondante si bien qu'il est devenu difficile de distinguer les différents courants et, plus important encore, d'identifier une position claire du XVIII ${ }^{\mathrm{e}}$ siècle par rapport au XVII ${ }^{\mathrm{e}}$ siècle 
et au $\mathrm{XIX}^{\mathrm{e}}$ siècle. La référence au « long dix-huitième siècle » est d'usage courant en Grande-Bretagne, dans la mesure où l'étude de cette période s'étend à la fois à la deuxième moitié du XVII ${ }^{\mathrm{e}}$ siècle et à la première moitié du XIX ${ }^{\mathrm{e}}$ siècle. Le problème avec cette périodisation est qu'elle nous fait perdre le sens et la mesure du changement. Le XVIII ${ }^{\mathrm{e}}$ siècle a toujours refusé d'être perçu comme un «pas vers la modernité » et a revendiqué une identité propre. Ce faisant, le danger est grand de se retrouver isolé.

\section{Leora Auslander}

S'il s'agit de distinguer des objets d'études plutôt que des périodisations, la distinction reste utile. Je vois deux avantages à comprendre la seconde approche comme celle qui met l'accent sur le « quotidien » et la « culture matérielle » par opposition à la société de consommation (et non pas comme une approche qui se concentre sur la longue durée plutôt que sur le temps court, ce qui est une autre discussion). D'abord, cela oblige le chercheur à aborder l'étude des objets et de leur sens potentiels avec un esprit ouvert. Alors que le travail généalogique est évidemment important, il est tout aussi crucial que les historiens essaient de penser le passé dans ses propres termes. Les objets n'ont pas toujours eu le même rôle et la même place au fil du temps. Si l'on se limite à vouloir comprendre l'origine de notre rapport actuel aux choses, on risque de passer à côté de ce qui est intéressant et important au sujet du passé. On s'expose également à des risques supplémentaires d'anachronisme. C'est une erreur de croire que l'acquisition et la mise en scène des objets du quotidien sont systématiquement les lieux où se forment les identités individuelles et collectives ou le sens social. Ensuite, et cette remarque découle de la précédente, l'approche par la culture matérielle évite la quête apparemment incessante pour dater toujours plus tôt le début de la « société de consommation » ou même de la « consommation de masse ». Quand on en vient à définir le $\mathrm{XV}^{\mathrm{e}}$ siècle comme une période caractérisée par la consommation de masse, on peut considérer que le concept a perdu tout son sens. Ceci étant dit, la transformation du XVIII ${ }^{\mathrm{e}}$ siècle a été d'une importance cruciale tant elle a façonné notre compréhension de la modernité.

Dans vos travaux, vous avez utilisé et problématisé la figure du consommateur ainsi que les notions de marché et de consommation. Quelles définitions en donnez-vous? Certains historiens ont proposé la thèse d'une révolution de la consommation au XVIII siècle, qu'en pensez-vous? 


\section{Giorgio Riello}

Je suis très heureux que cette question soit posée dans la mesure où je réalise que les historiens se réfèrent à des définitions très différentes. La remarque est valable pour moi qui ai utilisé des notions assez variées de consommateur, consommation et de marché en fonction des besoins. Il est frappant qu'à la différence des débats des années 1970 et 1980 sur la proto-industrialisation, la discussion sur la société de consommation au $\mathrm{XVIII}^{\mathrm{e}}$ siècle n'a pas prêté beaucoup attention aux définitions des concepts qui sont justement au coeur du débat. Deux raisons peuvent expliquer cette situation : d'abord, la discipline qui aurait raisonnablement pu fournir des définitions et des modèles - l'économie - s'est avérée plutôt inutile pour toutes les questions liées à la consommation. Dans son ensemble, l'histoire économique continue de tenir l'histoire de la consommation pour quelque chose de suspect. C'est pourquoi nous disposons davantage de bons exemples d'histoires sociales et culturelles du consommateur, du marché et de la consommation plutôt que d'histoires économiques. Ensuite, l'imprécision des définitions s'explique par le fait que les études portant sur la consommation ont été conçues de façon ascendante, en partant des archives pour créer un tableau général plutôt que le contraire. Il s'agit là bien sûr d'une généralisation, dans la mesure où ces discussions ont aussi mobilisé des chercheurs comme Jan de Vries, l'un des historiens économiques les plus importants de sa génération, doté d'une solide formation économique et par là même intéressé par l'élaboration de modèles. $\mathrm{La}$ « révolution industrieuse » proposée par de Vries - un concept emprunté à l'histoire économique japonaise - a l'ambition de rassembler le travail et les comportements du consommateur en soutenant qu'au cours des trois derniers siècles c'est l'intensification du travail affecté à la production de biens destinés à être vendus sur le marché qui a permis aux gens de pouvoir consommer davantage. Travailler plus pour consommer plus : rien de plus vrai dans la société d'aujourd'hui. Mais le modèle proposé par de Vries est aussi une réponse à l'idée de « révolution de la consommation » comme changement radical dans la taille, la nature et l'impact de la consommation sur la vie des gens au cours du XVIII ${ }^{\mathrm{e}}$ siècle. Personnellement, je trouve le concept utile quand il s'agit d'enseigner aux étudiants de première année. Mais si l'on considère les travaux des principaux participants à ces débats la sainte trinité McKendrick, Porter et Brewer, mais aussi Weatherill et Berg pour l'Angleterre, Shammas et Breen pour l'Amérique, Shamma et Blondé pour les Pays-Bas, Yun-Casalilla pour l'Espagne, etc - on s'aperçoit qu'il 
faudrait plutôt parler d' « évolutions de la consommation » caractérisées par différentes chronologies du changement.

\section{Colin Jones}

C'est à McKendrick, dans les années 1970 et 1980, que l'on doit l'idée d'une révolution de la consommation, à une époque où il était encore à la mode chez les historiens de découvrir des révolutions dans tous les aspects possibles et imaginables du passé. Le mot fait encore l'objet d'un emploi abusif et le moins que l'on puisse espérer des discussions actuelles serait qu'en émerge une terminologie analytique plus sobre et plus utile. Les changements dans le comportement des consommateurs sont certainement assez évidents pour justifier l'attention des chercheurs, notamment en France et dans le monde anglo-saxon. Pourtant, là aussi, l'échelle et la pénétration du marché, surtout en dehors des milieux urbains, doivent être débattus, comme Leora Auslander l'a récemment montré. Par ailleurs on peut détecter des changements notables dans le comportement des consommateurs dans d'autres sociétés plus éloignées dans le temps (l'Italie de la Renaissance, par exemple, comme l'ont montré les travaux d'Evelyn Welch $)^{7}$. Il faudrait probablement recommander d'éviter l'expression « révolution de la consommation » jusqu'à ce que l'idée générale du comportement des consommateurs au cours de la période moderne ait été définie de façon plus précise.

\section{Leora Auslander}

Les recherches menées ces trente dernières années ont démontré l'existence d'une augmentation de la quantité de biens disponibles, de même qu'un changement de leur nature, de leur prix et de leur sens au cours du XVIII ${ }^{e}$ siècle dans l'ensemble du monde atlantique. Je ne suis toutefois pas convaincue que l'expression « révolution de la consommation » est adéquate pour définir correctement ces transformations. Une telle révolution impliquerait une modification de la signification des objets à un point tel que l'identité individuelle et collective de la plupart des gens en viendraient à découler prioritairement des produits qu'ils achètent. J'avancerais cependant que - même si l'on ne peut dire ni pour l'Amérique coloniale ni pour la France du XVIII ${ }^{\mathrm{e}}$ siècle que la société de consommation s'y soit développée au point de façonner les identités individuelles et

(7) Evelyn WELCH, Shopping in the Renaisance: Consumer Culture in Italy, 1400-1600, Londres, 2005. 
collectives - l'accessibilité des biens de consommation et la publicité de plus en plus sophistiquée utilisée pour les vendre, ont rendu possible une nouvelle façon de penser la place des objets dans la production du sens social et du changement politique. Cette position suppose implicitement qu'un « consommateur » n'est pas simplement une personne qui acquiert et utilise des biens ; dans ce cas tout le monde est un consommateur. On ne peut être un consommateur qu'à partir du moment où l'on dispose d'un accès économique à une variété de biens symboliquement riches, librement consommables sans lois somptuaires ou toute autre forme de régulation, ainsi qu'à des informations sur ces produits (sous la forme de publicités, de conseils et d'exposition), de façon à permettre à l'individu de faire des choix raisonnablement libres sur les biens qu'il acquière.

\section{Daniel Roche}

Je ne crois pas avoir eu une utilisation originale de la notion de marché, autre que le lieu de la réalisation des échanges. Le développement commercial du XVIII ${ }^{\mathrm{e}}$ siècle a entraîné une extension des transactions et une transformation des marchandises. Dans la ville, les différents aspects de ces changements sont fondamentaux, car ils conduisent à s'intéresser à la hiérarchie et à la fonction des lieux et des modes de l'échange. La circulation des marchandises où se jouent les transformations de la consommation passe par les boutiques qui acquièrent une nouvelle visibilité, le shopping, mais aussi par les formes de la revente, le regrat alimentaire, le fripier, le neuf et l'usé, ont place dans la vente, mais autrement aussi, le don et le vol. Ce qui justifie que l'on doit replacer les controverses économiques sur la liberté et le contrôle dans une perspective élargie, ouverte à la figure des consommateurs et à l'analyse des comportements individuels révélés dans l'étude des choix et des goûts, soit encore par l'examen des relations sociales qui leur donnent sens. Une autre définition du marché est peut-être nécessaire pour ne pas projeter l'interprétation standard résultant de la définition par la décision libre des choix individualisés et des offres autonomes. C'est un marché qui se dévoile dans la société des consommateurs de l'Ancien Régime avec des niveaux d'implication diversement développés. Beaucoup de choses restent à étudier dans les campagnes et dans les villes pour en comprendre le fonctionnement, soit les actions et les réseaux des individus, des forces culturelles et politiques, donc des normes explicites ou implicites qui encadrent les consommations. Pour dire bref et donner un seul exemple, on n'a toujours pas prouvé l'action efficace des lois somptuaires. S'il y a une révolution de la consommation, elle s'est faite progressivement, mais avec une avancée urbaine décisive 
comme l'a montré Jean-Claude Perrot à Caen et d'autres ailleurs. La société des consommateurs fonctionne sans doute simultanément à l'économie marchande, à l'économie non marchande et à l'économie non monétaire comme l'a écrit Polanyi. La consommation vise à la fois le gain, le profit, mais aussi les relations réciproques, la vie matérielle et les réseaux sociaux qu'elles relient.

Dans l'histoire des révolutions politiques du XVIII siècle, le boycott, l'encadrement des prix (notamment la question du maximum) et le refus des taxations apparaissent comme des moments clefs, parfois déclencheurs, notamment en France et dans l'Angleterre coloniale. Peut-on dire que le phénomène nouveau de la consommation a préparé, voire provoqué les révolutions politiques en France et sur le continent américain? Ou au contraire, ce phénomène est-il cantonné à la sphère domestique et privée ?

\section{Leora Auslender}

Les nouvelles idées - comme celle qui veut que les objets possédés par les gens ordinaires aient un sens symbolique et affectif ; ou celle qui suppose que les gens sont liés les uns aux autres par les styles et les goûts qu'ils partagent - générées par le développement de la consommation ont bel et bien créé la base essentielle aux réflexions révolutionnaires sur la place de la culture matérielle dans la transformation politique. J'irais jusqu'à soutenir que les transformations de la culture marchande au $\mathrm{XVIII}{ }^{\mathrm{e}}$ siècle ont joué un rôle capital dans la forme prise par les révolutions américaine et française et ce faisant leur impact ne s'est certainement pas limité à la sphère domestique ou à la sphère privée. Le fait qu'il existe de nombreuses sources textuelles et iconographiques qui témoignent des usages explicitement politiques de la culture matérielle, indique que pour les acteurs des révolutions atlantiques de la fin du XVIII ${ }^{e}$ siècle les objets avaient leur importance - même les choses les plus banales avaient leur place dans la transformation révolutionnaire. Des efforts ont été faits pour révolutionner l'habillement, les formes architecturales, et la vaisselle (entre autres choses). Qu'il s'agisse d'obtenir l'indépendance, ou de faire tomber la monarchie et créer une république, les révolutionnaires de l'aire atlantique de la fin du XVIII ${ }^{\mathrm{e}}$ siècle ont cru dans le pouvoir pédagogique et figuratif de la culture matérielle.

La politisation du vêtement dans les révolutions américaine et française en est un exemple saillant. De part et d'autre de l'Atlantique, les acteurs politiques étaient convaincus de l'importance de l'habillement 
dans la dynamique transformatrice ; mais il existe aussi des différences significatives entre les deux contextes. Élément clé du vestiaire révolutionnaire américain, le homespun est un vêtement qui a pour caractéristiques essentielles d'être réalisé au sein du foyer ou sur la propriété de celui qui le porte, à partir d'une pièce de tissu produite sur place, dans les colonies américaines et non importé d'Angleterre. Il s'agit donc d'un produit domestique dans les deux sens du terme, au sens social, à la maison, c'est-à-dire chez soi, et à la maison au sens politique, c'est à dire sur le continent américain. Le vêtement acquiert ainsi une signification particulière pour celui qui le porte : il englobe tout à la fois l'idée d'indépendance, d'autonomie et de maturité politique. Le pantalon emblématique des sans-culottes est quant à lui un vêtement traditionnellement porté par les hommes des classes laborieuses (même s'il se trouve des artisans en milieu urbain qui s'en accommodent). Porté avant la Révolution, il signale immédiatement l'appartenance sociale de son propriétaire. Au cours de la Révolution, le port délibéré de ce vêtement devient un signe d'appartenance politique et non plus de classe. Et il est tout à fait révélateur que dans la Révolution américaine, le vêtement incarne la nation qui n'a pas encore d'existence concrète, tandis qu'en France, il devient le symbole d'une classe sociale (ou un groupe si l'on préfère éviter la référence aux classes) privée du pouvoir politique. L'étude de la mode vestimentaire éclaire alors les enjeux fondamentaux de chaque révolution.

Deux autres choses distinguent encore le homespun américain du pantalon des sans-culottes. Premièrement, notons que si des deux côtés de l'Atlantique les hommes comme les femmes utilisent les vêtements à des fins politiques, dans le cas français il s'agit d'un vêtement par définition masculin. Il n'existe pas d'équivalent féminin au pantalon des sans-culottes, c'est à dire un type de vêtement directement associé aux femmes des milieux populaires. Celles qui souhaitent être perçues comme sans-culottes se contentent simplement de porter des vêtements modestes. À l'inverse, dans la mesure où le homespun fait davantage référence à un mode de fabrication qu'à un type de vêtement particulier, il est adopté aussi bien par les hommes que par les femmes.

Deuxièmement, alors que dans la France du XVIII ${ }^{\mathrm{e}}$ siècle comme dans l'Amérique coloniale le tissage est une activité essentiellement féminine et que la production de vêtements par les foyers ruraux est importante, l'habit des sans-culottes n'est pas associé au foyer familial, ni aux femmes ni à la maison. À l'inverse, ces associations sont fortes pour le homespun. Le boycott des produits manufacturés venus d'Angleterre et leur remplacement par des produits américains, stratégie politique fondamentale 
de la Révolution américaine, a été essentiellement mis en œuvre par les femmes. Dans l'Amérique de la fin du XVIII ${ }^{\mathrm{e}}$ siècle, les femmes sont à la fois consommatrices (elles constituent la plus grande partie de la demande de produits de consommation) et - du moins dans certaines régions - productrices de produits domestiques. Lorsque l'appel au boycott des étoffes venues d'Angleterre fut lancé, on a attendu des femmes qu'elles s'abstiennent d'acheter des tissus importés. Pendant les années de guerre, on leur a aussi demandé d'augmenter significativement leur production de vêtements, non seulement ceux destinés à leur usage personnel, mais également pour fournir l'armée. En Amérique, l'imbrication particulière du public et du privé, l'importance de la maison, à la fois au sens littéral et politique, le rôle central du mouvement de boycott (et par conséquent de l'importance accordée à la production domestique) et enfin l'importance des femmes dans la production et la consommation de produits textiles, a conféré à ces dernières une place politique singulière dont on a pu mesurer l'effet à long terme.

Explorer la Révolution américaine au miroir de l'expérience française à l'aide du homespun et du pantalon des sans-culottes permet de complexifier les idées reçues sur les différences nationales et d'enrichir notre compréhension de chaque processus révolutionnaire. Même si les révolutionnaires puis les citoyens américains se sont montrés beaucoup plus timides que les Français sur la question du pouvoir de l'État et de ses domaines d'intervention, la politisation du foyer a été en fait beaucoup plus intensive et étendue aux États-Unis qu'elle ne l'a été en France. Privées de la participation politique formelle, les femmes américaines n'en ont pas moins conservé un sens aigu de leur rôle et de leur capacité d'action sur le plan national. En France, la politisation de l'espace public fut totale, mais l'engagement de la Révolution à l'égard de la liberté et la définition qu'elle en proposait excluaient le foyer familial de la vie politique et par conséquent les femmes dont la maison était considérée comme un abri.

\section{Colin Jones}

Dans mon article « The Great Chain of Buying », paru en 1996 dans l'American Historical Review, j'avais cherché à montrer comment le marché (saisi à travers le monde de la publicité publiée dans les journaux) en produisant une vie publique plus harmonieuse et de nouvelles formes de citoyenneté, favorise et contribue à façonner de nouvelles manières d'appréhender l'identité sociale ainsi qu'une vision neuve et optimiste de la place du marché. Timothy Breen a proposé une interprétation similaire 
concernant la Révolution américaine ${ }^{8}$. Pour une grande majorité d'entreeux, les révolutionnaires de 1789 ont été formés par le marché ; on pourrait d'ailleurs utilement appliquer cette idée de marché aux Lumières comprises comme un marché des idées. La France de 1789 comptait davantage de révolutionnaires « consommateurs », favorables au marché, que l'inverse. En général, les historiens n'ont pas perçu à quel point l'engagement politique révolutionnaire s'est accompagné de l'acceptation du marché bien plus que de sa critique ou de son rejet. On en a fait plus qu'il n'aurait fallu sur la politique de la vertu d'un Robespierre, dont les opinions sur le sujet étaient pourtant loin d'être représentatives et sont parfois même contradictoires (l'apôtre de la vertu républicaine n'était-il pas lui-même un gros consommateur de poudre à cheveux ?). En 1999, dans un article écrit avec Rebecca Spang, nous avons cherché à montrer combien même les sansculottes de l'an II se sont adaptés aux nouvelles normes de consommation'. La liste des produits dont le prix fut fixé par la loi du Maximum n'a rien de spartiate, et témoigne de l'immense extension du marché au cours du siècle. Les denrées alimentaires autrefois luxueuses sont devenues des nécessités pour les masses populaires de l'an II. Au grand dam de Robespierre, les sans-culottes ont manifesté autant pour réclamer du sucre que pour réclamer du pain ${ }^{10}$. L'économie morale est un concept à géométrie variable. Il peut être utile de garder ceci en tête lorsque que l'on discute des formes de résistance comme le contrôle des prix et la taxation populaire, qui déjà à l'époque pouvaient se targuer d'une histoire longue, entamée bien avant la montée en flèche de la demande des consommateurs à la fin du XVIII ${ }^{\mathrm{e}}$ siècle $^{11}$.

Enfin, mais faut-il le préciser, la conséquence politique d'une place plus importante au sein du marché diffère en fonction du contexte. Les conséquences postulées plus haut pour la France et l'Amérique sont bien différentes dans le cas de l'Angleterre. Il serait également utile de prendre en compte le rapport entre le comportement des consommateurs et les

(8) Timothy H. BREEN, The Market-Place of Revolution : How Consumer Politics Shaped American Independence, Oxford, 2004.

(9) Colin JONES \& Rebecca SPANG, «Sans-culottes, sans café, sans tabac : shifting realms of luxury and necessity in eighteenth century France », in Maxine Berg \& Helen Clifford (eds), Consumer and luxury : consumer culture in Europe, 1650-1850, Manchester, 1999.

(10) Robespierre : «Le peuple de Paris doit se lever non pour recueillir du sucre mais pour terrasser les brigands ». Discours à la Convention, 25 février 1793, au moment où la hausse du prix du sucre est à l'origine d'émeutes dirigées contre les épiciers.

(11) Sur ce sujet, voir les travaux classiques d' E.P Thompson, George Rudé, Richard Cobb et Eric Hobsbawm qui tous soulignent la dimension traditionnelle des formes de contestation liées à l'économie morale. 
attitudes politiques en dehors du contexte anglo-américano-francais au sein duquel la plupart des travaux dans ce domaine semblent avoir été écrits. À l'heure actuelle, les autres historiographies nationales ne semblent pas faire beaucoup de cas du phénomène consumériste (malgré les facteurs de la globalisation discutés plus bas).

\section{Giorgio Riello}

J'ai dû aller vérifier les deux étagères qui dans mon bureau sont dédiées à l'histoire de la consommation. Je suis désolé mais je ne partage pas l'opinion de mes collègues sur le sujet. J'inclus tout ce qui a été dit au sujet de l'excellent travail de Timothy Breen pour l'Amérique coloniale, l'idéologie du homespun de Michael Zakim, les recherches menées par Colin et Leora, mais aussi par Rebecca Sprang, Richard Wrigley et Ulrich Lehman. Et malgré ce qui apparaît comme la preuve indéniable que les chercheurs ont travaillé dur pour mettre en évidence le rapport entre consommation et révolutions politiques, je pense que la démonstration est loin d'être convaincante et ce pour deux raisons. D'abord, la « consommation » a-telle « préparé » ou « provoqué » les révolutions ? Je vais peut-être passer pour un chercheur tatillon, mais je ne crois pas que l'on puisse mettre en évidence de corrélation claire. On a le sentiment que ce qui s'est produit dans la «sphère du consommateur » allait dans le même sens que ce qui était en train de se passer dans la sphère politique, le marché allant jusqu'à reprendre à son compte le discours politique dominant, mais j'ai bien peur que dans leur grande majorité les gens qui vivaient à cette époque n'étaient pas mus par la consommation et que celle-ci n'a joué qu'un rôle mineur dans la constitution de leurs idéaux et la mise en forme de leurs actions. Ensuite, et c'est peut-être le point le plus important, j'ai réalisé en faisant cet exercice de vérification dans mes étagères que les travaux évoqués plus haut se trouvent au beau milieu d'un océan de publications sur la consommation qui ne s'intéressent en aucune manière au rapport entre les révolutions politiques et la consommation au XVIII ${ }^{\mathrm{e}}$ siècle. Leurs auteurs semblent plus préoccupés par la consommation de thé et les scènes de conversation (qui dépeignent généralement la vie des familles aisées en train de prendre le thé) que par la Boston tea party. Et je ne parle pas des travaux sur la mode, les commodités, la décoration d'intérieur, le luxe etc. : tous font la part belle à la sphère domestique et privée mais aussi à la question de la sociabilité et à la problématique des interactions sociales qui se développent autour de la consommation (du shopping à l'échange de politesses, en passant par les cures thermales à Tumbridge Wells et à Bath). J'ai le sentiment que nous avons créé un consommateur 
moyen du XVIII ${ }^{\mathrm{e}}$ siècle un peu trop semblable à notre propre expérience et à notre propre identité : un personnage plutôt civilisé mais fainéant, davantage préoccupé par le type de boucle à porter avec telle ou telle paire de chaussures que par le destin du monde. L'histoire de la consommation est aussi très féminine, il semble que nous en sachions beaucoup plus sur le rapport des femmes à la consommation que sur celui des hommes.

\section{Daniel Roche}

L'intérêt pour la réaction à tout ce qui concerne les prix, l'encadrement du marché, ne peut se démentir dans l'étude des révolutions, au $\mathrm{XVIII}^{\mathrm{e}}$ siècle, après. Le refus des taxations est toutefois à replacer dans une perspective qui a été développée par Dominique Margairaz et Philippe Minard $^{12}$. Le contrôle des prix qui a survécu à la Révolution française est une traduction de l'Économie morale de la foule, analysée par E.P. Thompson, et Steve Kaplan en a montré de façon quasiment définitive les implications, même si celles-ci ont été nuancées dans le long terme. Ce qui est à retrouver, c'est la diversité qui est à l'œuvre dans le mouvement divers des taxes urbaines, de la surveillance des prix, du contrôle des marchés et des marchands, du Maximum, et aussi ce que visent les consommateurs et leurs représentants. Le phénomène de la consommation n'a pas le même sens selon l'articulation des consommateurs au marché, selon les produits. Les troubles de la Révolution et l'élargissement des exigences qu'ils révèlent en matière de taxe montrent aussi l'élargissement des consommations, le savon, le sucre, le coton, par exemple, en même temps que la diversité des dispositifs de contrôle. L'histoire du Maximum imposé par les circonstances montre comment la consommation étendue à tous les biens, généralisée à toutes les catégories sociales a participé au passage de l'économie morale à l'économie nouvelle où la circulation est l'âme du commerce. C'est peut-être là que la sphère domestique, résultant de l'industrious revolution, la révolution de la consommation qu'on peut lire dans l'histoire des budgets (présentée dans l'Histoire des choses banales) rencontre les exigences collectives. La consommation élargie fait que la protestation ne vise plus seulement la défense pour la survie à l'œuvre dans les émeutes de la faim, mais le maintien d'un niveau de vie et d'une consommation variée. Là réside peut-être l'importance décisive de la transformation des mœurs du XVIII ${ }^{\mathrm{e}}$ siècle.

(12) Dominique MARgaIRAZ et Philippe MinARD, « Marché des subsistances et économie morale : ce que " taxer" veut dire », $A H R F$, avril-juin 2008, n 352, p. 53-99. 
Le commerce apparaît aussi comme un catalyseur du changement politique, un accélérateur des temporalités. Peut-on dire que le commerce et l'économie atlantiques ont favorisé l'exportation de la révolution sur le continent américain (dans l'Angleterre coloniale, à Saint-Domingue, mais aussi dans les colonies latino-américaines au début du XIX ${ }^{e}$ siècle) ? Plus généralement, comment l'histoire globale, en décentrant le regard, a-t-elle permis de changer le point de vue sur les révolutions du XVIII siècle, entendues au sens large?

\section{Colin Jones}

La thèse de la Révolution atlantique, prêchée dans les années 1950 et 1960 par Jacques Godechot et l'historien américain Robert R. Palmer, cherchait à mettre les révolutions française et américaine au centre d'un enchaînement de révolutions supposées « démocratiques » de part et d'autre de l'Atlantique au cours de la seconde moitié du XVIII ${ }^{\mathrm{e}}$ siècle $^{13}$. À l'époque, Godechot et Palmer se sont retrouvés confrontés à un torrent de critiques, ils furent accusés notamment de s'en prendre - sacrilège - au statut de la Révolution française, notre mère à tous, et leurs arguments furent jugés comme une tentative idéologique visant à légitimer l'existence de l'OTAN ! Certes, la nature soi-disant « démocratique » de bien des soulèvements de la fin du XVIII ${ }^{\mathrm{e}}$ siècle pourrait être questionnée, et les aspects téléologiques de l'hypothèse Palmer-Godechot en faveur du bloc de l'Ouest apparaissent aujourd'hui extrêmement datés. Pourtant, ces dernières années, quelque chose qui ressemble beaucoup à la thèse de la Révolution atlantique a de toute évidence fait un retour, notamment, mais pas uniquement, chez les chercheurs anglais et américains. L'ouvrage dirigé récemment par David Armitage et Sanjay Subrahmanyam, The Age of Revolutions in Global Context, 1760-1840, témoigne du chemin parcouru depuis Godechot et Palmer, mais souligne également qu'il reste beaucoup à faire : le chapitre sur la Révolution française brillamment rédigé par Lynn Hunt montre combien il est toujours difficile de penser la Révolution française comme un phénomène transnational. «L'âge des révolutions » ne s'est pas arrêté en 1799 (comme Palmer le pensait), il s'étend à la fois à la Révolution haïtienne et aux révolutions de l'Amérique espagnole au début du XIX ${ }^{\mathrm{e}}$ siècle. Les auteurs qui ont contribué à l'ouvrage dirigé par Armitage et Subrahmanyam élargissent la perspective à la fois dans l'espace et dans le temps, en

(13) Jacques GODECHOT et Robert R. PALMER, « Le problème de l'Atlantique du XVIII ${ }^{\mathrm{e}}$ au XX $\mathrm{XX}^{\mathrm{e}}$ siècle », Comitato internazionale di scienze storiche. Congresso internazionale di Scienze storiche, Roma, 4-11 Settembre 1955, Relazioni 5 (Storia contemporanea), Firenze, 1955. 
travaillant dans un cadre véritablement mondial. Cet élargissement se traduit notamment par un regain d'intérêt des historiens pour l'histoire du sous-continent indien. L'idée d'une interconnexion globalisée, rendue perceptible par l'étude fine de la production et de la consommation de biens manufacturés, semble indiquer que la quête pour trouver des points de convergence entre le comportement des consommateurs au long d'un $\mathrm{XVIII}^{\mathrm{e}}$ siècle et l'existence d'un mouvement politique de transformation de la société dont l'impact est évident bien après 1800, est de nouveau d'actualité.

\section{Leora Auslander}

Penser en terme de monde atlantique plutôt que de se concentrer sur les histoires nationales change fondamentalement la perception des dynamiques à l'œuvre dans ces premières révolutions modernes. Compte tenu que l'interconnexion économique est essentielle au développement de la société marchande et que ce développement est lui à l'origine des recontextualisations de l'ordre social qui sous-tendent les soulèvements politiques, ces révolutions nous apparaissent maintenant définitivement pour le moins « atlantiques », sinon « globales », par nature. Par exemple, les Africains réduits à l'esclavage ont traversé l'Atlantique pour travailler en Amérique du Nord et ce faisant, par leur travail, ils ont influencé les formes culturelles de cette société. Les colons venus d'Angleterre, de France, des pays germaniques et des Pays-Bas ont certes commencé de nouvelles vies en Amérique du Nord, mais ils n'en ont pas moins conservé des liens avec l'Europe. Les livres, le thé, la porcelaine, les étoffes, les miroirs et les partitions de musique ont aussi traversé l'océan. L'histoire du commerce et des révolutions du XVIII ${ }^{\mathrm{e}}$ siècle est ainsi fondamentalement et nécessairement une histoire «atlantique » dans le sens qu'il s'agit d'une histoire rendue possible par les mouvements et l'interconnexion des hommes, des biens et des idées permis par cet espace maritime.

\section{Giorgio Riello}

On peut aussi comprendre le développement du commerce comme le signe de l'interconnexion croissante du monde au XVIII ${ }^{\mathrm{e}}$ siècle. Et bien évidemment les marchands n'amènent pas avec eux que des biens de consommation. L'Atlantique est ainsi un bon exemple de création de structures de longue distance qui soutiennent un système économique nouveau, à la fois par son échelle et par sa portée. Les colonies sont synonymes de réserves ou de ressources (naturelles mais aussi agricoles comme c'est le cas avec le sucre, le cacao ou le coton) tandis que les colons 
(et leurs subordonnés, esclaves ou paysans libres sous contrat) forment le marché. Ce récit européo-centré est valable à la fois pour l'Amérique du Nord française et anglaise et pour l'Amérique du Sud espagnole et portugaise. Il montre toutefois combien les intérêts de la métropole peuvent être différents de ceux des colons. Des tensions s'ensuivent. Mais il y a encore deux autres aspects à cette question. Le premier est la guerre plutôt que la révolution. La Guerre de Sept Ans est un des plus grands rééquilibrages du pouvoir global au XVIII ${ }^{\mathrm{e}}$ siècle. Elle éclate au milieu du siècle, c'est un tournant fondamental dont les répercussions affectent non seulement l'Europe et l'Amérique du Nord mais également l'Asie. Et ceci nous amène au deuxième point de votre question au sujet de la perspective globale. Le commerce avec l'Asie consistait principalement à faire venir des produits manufacturés (des cotonnades en particulier). Il a conduit à une expérience coloniale d'un autre type. On trouve dans le livre dirigé par Armitage et Subrahmanyam une carte intéressante qui rappelle l'existence au niveau mondial d'un grand nombre de révoltes majeures, de guerres et de révolutions qui n'ont rien à voir, sinon pas grand-chose, avec les puissances européennes ou l'expansion commerciale du continent. Elles font néanmoins partie d'une reconfiguration globale de la politique mondiale au XVIII ${ }^{\mathrm{e}}$ siècle.

$\grave{A}$ distance des pratiques de consommation et de la structuration des marchés, quelles sont les lectures savantes et juridiques de cette économie politique et de l'articulation entre marché, régulations économiques et ordre social au XVIII e siècle ? Les révolutions de la fin du XVIII siècle ont-elles fait évoluer les conceptions de l'économie morale et politique ; en d'autres termes, assiste-t-on à une rupture ou à une affirmation du modèle libéral du marché en Europe, mais aussi dans les espaces extra-européens?

\section{Giorgio Riello}

L'idée d'un XIX ${ }^{\mathrm{e}}$ siècle libéral tout droit sorti des soulèvements révolutionnaires européens reste encore très confuse. L'opposition entre le monde économique libéral du XIX ${ }^{\mathrm{e}}$ siècle et l'économie protectionniste, mercantile et fortement régulée du XVIII ${ }^{\mathrm{e}}$ siècle est aujourd'hui remise en cause. Du point de vue du libre échange, l'Angleterre est la seule nation européenne ayant embrassé activement les doctrines économiques libérales et, malgré tout, les idées d'Adam Smith n'ont été véritablement mises en pratique qu'à l'horizon des années 1840. Nous devons nous rappeler également que parmi les nombreuses personnalités farouchement 
opposées aux idées smithiennes se trouvaient des hommes comme Frédéric List, Alexander Hamilton et les Francais Chaptal et Dupin. Au-delà du problème du libre échange, le monde libéral s'impose comme celui de la libre entreprise au sein duquel émergent la firme moderne, la responsabilité limitée et les flux de capitaux importants gérés par de grands établissements bancaires. Les structures sont aussi posées - financières d'une part, mais aussi celles qui permettent les communications et les déplacements, pensons simplement au télégraphe, au téléphone, aux voies ferrées - pour connecter entre elles les différentes régions du monde. Williamson et O'Rourke voient le $\mathrm{XIX}^{\mathrm{e}}$ siècle comme la première phase de globalisation véritable avec l'intégration des marchés mondiaux et la convergence du prix des matières premières. Cependant, tout ceci ne nous dit pas ce qui l'a provoquée. Je vois le modèle libéral non pas tant comme le fruit des révolutions mais comme celui de la Restauration qui a installé l'Angleterre fermement au sommet du système. Il était dans l'intérêt de celle-ci et plus tard des États-Unis d'ouvrir les marchés et de faire pression pour imposer le libre échange et la mobilité du capital et du travail. Le travail de Philippe Minard sur le colbertisme, de même que ses recherches plus récentes sur l'Angleterre, sont utiles car il y montre l'existence de différences importantes entre ces deux nations au XVIII ${ }^{\mathrm{e}}$ siècle, l'Angleterre s'engageant bien avant "l'ère des révolutions » sur le chemin - en terme de structures économiques, d'idées et de conditions - qui mène au monde libéral. Le drame de l'Europe continentale - et la cause du futur incertain de l'Union Européenne aujourd'hui, pourrait-on dire - est qu'elle n'a jamais adhéré pleinement aux idées libérales. Le corporatisme et la régulation dans la France, l'Italie ou même l'Allemagne d'aujourd'hui ne sont pas sans rappeler la régulation économique de l'Ancien Régime.

\section{Daniel Roche}

J'ai évoqué (question 3) le problème de l'économie morale, c'est une des lectures de l'économie politique et de la régulation économique. Elle ne se confond pas avec la régularisation du mercantilisme, avec sa volonté d'unification territoriale et administrative et aussi cette entreprise au service d'une politique, instrument de grandeur politique et militaire. Ce mercantilisme, c'est la théorie d'un système de production de richesse et non de distribution, dit Pierre Deyon, après Eli Filip Heckscher, et par comparaison avec le modèle libéral. On sait par les travaux de JeanPierre Hirsch sur le négoce, de Philippe Minard sur les inspecteurs des manufactures et les corporations, de Steven Kaplan sur le complexe frumentaire, les subsistances, le pouvoir et l'approvisionnement, ce qu'il contient. Ces études montrent bien comment fonctionnent jusqu'à la 
Révolution les systèmes traditionnels et comment ils évoluent face à la commercialisation, au développement technique, à la consommation. C'est aussi pour nous le moyen de découvrir la différence entre le marché tel que le définissent les pratiques des échanges comme lieu social contrôlé et le marché fondateur du libéralisme ouvert au libre jeu de la concurrence et qui ne s'incarne plus comme pour les blés dans des lieux fixes et surveillés. On sait quelle place le marché ancien accordait au Roi, à sa responsabilité de père nourricier, à son rôle pour équilibrer liberté et surveillance. Avec la Révolution, et au XIX ${ }^{\mathrm{e}}$ siècle, il reste de cette idée que les taxes et le contrôle garantissent la confiance mais elles n'engagent plus la responsabilité morale et la mission pastorale de l'État. L'individualisme l'a emporté sur l'organicisme et l'inégalité.

En aval, il me semble qu'on pourrait rouvrir le chantier en l'élargissant à l'ensemble des produits de consommation de la Révolution, en suivant l'institutionnalisation des théories dans les lois sur la propriété, dans l'instauration du pouvoir des nouvelles élites administratives, des notables qui incarnent le nouveau fonctionnement de la justice, dans le rapport de domination, dans la législation économique et les monopoles d'État. C'est ce qu'a esquissé S. Ayad-Bergougnioux dans sa thèse sur $l a$ Bourgeoisie de robe et l'esprit d'État.

En amont, un grand chantier d'histoire intellectuelle européenne est resté ouvert. Il consisterait à analyser, de l'Ancien Régime à la Révolution et au-delà, le rapport religion-économie, pratiques et théories. Si la liaison entre les croyances et les dogmes d'une part, l'économie d'autre part, a été parmi les préoccupations de Max Weber, on a rarement tenté d'analyser les effets du développement économique en reliant valeurs religieuses, normes et pratiques avec les analyses théoriques et les comportements. Par rapport à notre temps, c'est un moyen de se demander comment on est passé de l'économie religieuse, morale, à la religion de l'économie avec ses temples, ses prophètes, ses écritures.

À partir d'une historiographie sur le marché et les consommations, il semblerait que la réflexion porte désormais de plus en plus sur la culture matérielle et l'anthropologie du quotidien des révolutions, étudiée à partir des objets - y compris ceux qu'elles produisent, même les plus éphémères (objets portatifs ou décoratifs, d'actualité ou commémoratifs, vêtements, iconographies). Que nous apprend ce materialturn des révolutions? Dans quelle mesure les révolutions transforment-elles le rapport aux objets du quotidien mais aussi 
produisent-elles de nouveaux types d'objets, avec une iconographie et des usages spécifiques?

\section{Leora Auslander}

Dans le contexte français comme dans le contexte américain, l'habillement et les objets du quotidien occupent une place incontournable, à la fois parce qu'ils sont partie intégrante de la transformation révolutionnaire, mais aussi parce qu'ils sont utilisés comme support pour la célébrer ou la commémorer. En Amérique du Nord, les travaux de couture occupent une place particulièrement importante, aussi bien pendant la période révolutionnaire qu' après l'indépendance. Dans les foyers américains, la fabrication de courtepointes est une façon typique de célébrer la Révolution. Sur certaines sont reproduites des images patriotiques, si bien que les familles peuvent littéralement s'endormir au contact des symboles de la nation. Ces travaux commémorent aussi des événements importants de la Révolution. Le motif connu sous le nom de «The Burgoyne Surrounded» (L'encerclement de Burgoyne) a ainsi été spécialement créé pour célébrer la bataille de Saratoga (17 octobre 1777) au cours de laquelle les 6000 soldats britanniques conduits par le général John Burgoyne furent encerclés et défaits par l'armée américaine. La réalisation des courtepointes et des broderies est une activité qui très lente : ces symboles, ces visages et ces lieux chargés de significations politiques ont d'abord pris forme dans l'esprit des femmes avant d'être réalisés à la main, au sein du foyer, pendant de longs mois, voire de longues années. La réalisation et l'utilisation d'objets du quotidien s'imposent alors comme une modalité particulière de la formation de la conscience politique. La politisation qui en résulte s'est prolongée bien au delà du moment révolutionnaire.

Dans le cas américain, la politisation intense de la vie familiale et le rassemblement de femmes produisant et consommant volontairement des biens américains se sont poursuivis au cours du XIX ${ }^{\mathrm{e}}$ siècle. Le savoir-faire des femmes américaines s'est manifesté dans deux domaines : l'organisation des consommateurs et la réalisation d'ouvrages de couture et de décoration pour soutenir le mouvement abolitionniste et la lutte contre l'alcoolisme. Les abolitionnistes ont repris l'idée du boycott (cette fois des produits issus du travail des esclaves) et celle de la pédagogie du quotidien à travers l'ameublement de la maison avec des objets porteurs d'un message abolitionniste. Les motifs que l'on rencontre le plus souvent représentent l'intérieur d'un bateau négrier dans le but de donner à voir l'espace brutalement confiné consacré à la marchandise ; un esclave agenouillé levant ses mains enchaînées en implorant la liberté ; ou des esclaves 
épuisés sous des plantes tropicales. La plupart des objets sur lesquels ces images ont été reproduites étaient fabriqués à l'étranger. En Angleterre, Josiah Wedgwood a commencé à produire des camées abolitionnistes en porcelaine dès les années 1770 ; ses modèles ont été exportés vers l'Amérique du Nord où les motifs ont ensuite été copiés et distribués. Les femmes ont ainsi acheté des objets très divers, des broches pour leurs vêtements, des gravures pour leurs murs, des services à thé pour leur salon, des carafes pour leur service à petit-déjeuner et des pipes pour leurs maris, qui avaient pour particularité d'être agrémentés de textes et d'images antiesclavagistes. Utilisés et manipulés quotidiennement, ces objets venaient constamment rappeler à chacun le positionnement politique du foyer ; ils servaient en outre de modèle pour les objets que les femmes réalisaient chez elles.

Comme à l'époque de la Révolution, beaucoup de ces objets ont été fabriqués de façon artisanale, à la maison, par des femmes travaillant seules ou en groupe, à l'image de ce porte-aiguille brodé des années 18301850, conservé au Peabody Essex Museum et qui représente une esclave agenouillée libérée par une autre femme, transformant le célèbre slogan antiesclavagiste britannique « $\mathrm{Ne}$ suis-je pas un homme et un frère ? » en « $\mathrm{Ne}$ suis-je pas une femme et une soeur ?». La courtepointe réalisée en 1842 par les femmes de l'Indiana Yearly Meeting of the Anti-Slavery Friends of Clinton County, Ohio, et Wayne County, Indiana, après avoir été exclues de l'Indiana Yearly Meeting of Friends à cause du radicalisme de leurs positions abolitionnistes, est un exemple particulièrement spectaculaire d'effort collectif. Chaque femme a d'abord conçu un motif, qu'elle a ensuite cousu sur un carré signé. La courtepointe est le résultat de tous ces éléments uniques mais complémentaires, qui font de l'ouvrage une fois achevé une déclaration audacieuse de responsabilité individuelle et collective. Les femmes afro-américaines se sont elles aussi engagées dans des activités similaires; si bien que la fabrication de courtepointes est devenue un élément important de l'esthétique noire après l'émancipation. Certaines esclaves affranchies, comme Sarah Sedgwick, ont même réalisé des broderies pour célébrer leur propre liberté. Enfin, des femmes ont organisé des ventes d'objets afin de collecter des fonds pour la cause abolitionniste, à l'image des dix «women managers » de la conférence anti-esclavagiste de Philadelphie qui tous les ans à partir de 1834 ont sollicité des dons de meubles, de porcelaines, d'éventails, d'épingles à cheveux, d'argenterie et autres objets du quotidien, pour les vendre. Dans ce cas, les objets en eux-mêmes n'avaient aucune portée politique, seule la vente en avait. 


\section{Daniel Roche}

L'histoire des changements de comportement à l'époque des Révolutions est, à mes yeux, marquée par deux interrogations : celle de la rupture de l'ancien système de circulation des biens et du luxe, celle de la mobilité accélérée dans la dimension locale, nationale et internationale. On n'a pas porté l'attention qu'il mérite à l'effet de redistribution entraîné par la désorganisation du marché, les confiscations, la mise en vente aux enchères, voire la captation des biens par la violence. Ainsi dans le domaine de l'économie équestre, la vente des chevaux du Roi est un événement fondamental pour l'élevage, l'armée, la symbolique du pouvoir, qui n'a jamais été étudié à ma connaissance.

Le Material turn des révolutions interroge partout la culture des apparences révolutionnée. Où passent la nouvelle emblématique et l'affirmation des contradictions ? L'an II a été de ce point de vue un moment de rupture où les transformations théoriques venues d'en haut - le vêtement étant présenté comme le modèle de la régénération républicaine rencontrent les capacités d'accueil des populations. Les modèles vestimentaires antérieurs ne sont pas bouleversés, mais comme l'a montré Richard Cobb, des changements de détails apparaissent. Ils sont liés à la spécificité même des circuits vestimentaires de production et de consommation, au rôle du vêtement comme outil d'échange et de revente. C'est dans des détails majeurs, la cocarde, le bonnet rouge, le pantalon, la carmagnole, les sabots, qu'on va saisir la novation temporaire qu'illustreront certains sans culottes qui promeuvent l'idéal du bon citoyen. On le saisit dans les images et dans les recueils de mode comme le Journal de la mode et du goût qu'il faut confronter aux archives judiciaires, car elles enregistrent l'expression des valeurs et de leur écho. Leur usurpation, les déguisements, les jeux de masque qu'observent et contrôlent les policiers en révèlent la capacité politique entre le culte et la dénonciation. La stabilisation révolutionnaire s'accompagne, en tout cas après Thermidor, d'une intense réflexion sur le langage des signes et sur l'affirmation de l'autorité politique par la définition d'un costume officiel. Ceci au moment où la réaction à la Terreur a déclenché la réapparition et l'accélération des modes. Au total, en ce domaine, la Révolution met en valeur deux fonctions fondamentales du vêtement, paraître ou transparaître, révéler ou masquer, et l'on se doute que, de la capitale aux provinces, le mouvement n'a pas eu la même ampleur et des rythmes identiques. 


\section{Giorgio Riello}

En disant plus haut que l'histoire de la consommation ne s'intéresse pas à la politique, j'ai consciemment exclu la culture matérielle. D'abord, permettez-moi de dire que je vois le material turn comme découlant de l'idée de consommation. Sans doute, certains critiques pourraient dire que la culture matérielle est la dernière et la pire dégénérescence des études de la consommation. Sans être aussi acerbe, il est indéniable que les historiens sont parvenus à se glisser jusque dans les réserves des musées et se confrontent désormais à toutes sortes d'objets qui sont parfois curieux. Je voudrais vous donner un exemple : le musée Carnavalet à Paris. Au premier étage, le public se voit proposer une collection d'objets assez particulière. Il s'agit d'un reliquaire constitué des objets donnés par Madame Royale à Madame de Tourzel et à sa fille lorsque la famille royale fut emprisonnée au Temple. La collection, absolument fascinante, comprend des portraits de Louis XVI, de la reine, du dauphin et d'autres membres de la famille royale, mais aussi des médaillons avec des cheveux de Louis XVI, de la reine et du dauphin et même un morceau de filet fait par Marie-Antoinette à la Conciergerie. Il ne fait aucun doute que ces objets sont des faux, mais force est de constater qu'ainsi réunis ils traduisent mieux qu'aucun livre le sens du drame ordinaire qui s'est joué alors. Au rez-de-chaussée du musée une enseigne provenant du Palais-Royal est exposée ( Aux Trois Ordres », enseigne de marchand de cocardes de l'époque révolutionnaire - inv. EN 120). Elle témoigne à sa façon de l'aspect mercantile de la Révolution et en particulier de sa tentative pour réformer la consommation et la mode, un sujet qui a reçu beaucoup d'attention ces dernières années. Ces objets (y compris une impressionnante et effrayante tête en cire de Louis XVI bizarrement agrémentée de quelques cheveux douteux) doivent nous amener, sinon à de nouvelles interprétations, du moins à envisager de nouvelles manières d'aborder des sujets bien connus et parfois banals. 
Les traductions des réponses de Leora Auslander, Colin Jones et Giorgio Riello ont été assurées par Julien Louvrier.

\author{
Leora AUSLANDER \\ lausland@uchicago.edu \\ Charlotte GUICHARD \\ charlotte.guichard@wanadoo.fr \\ Colin JONES \\ c.d.h.jones@qmul.ac.uk \\ Giorgio RIELLO \\ g.riello@warwick.ac.uk \\ Daniel RocHE
} Francoise.malvaud@college-de-france.fr 